\title{
MOOSE \\ Implementation of MAMBA
}

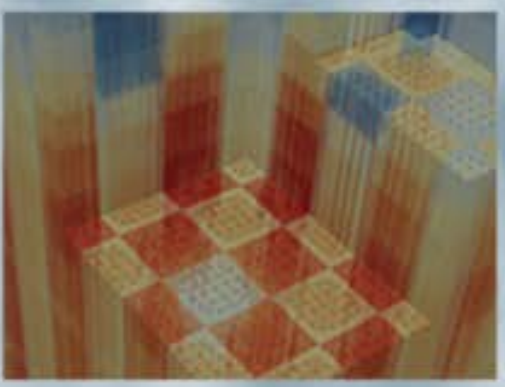

Christopher Matthews, LANL

Jack Galloway, LANL

January 17, 2017

Approved for Public Release
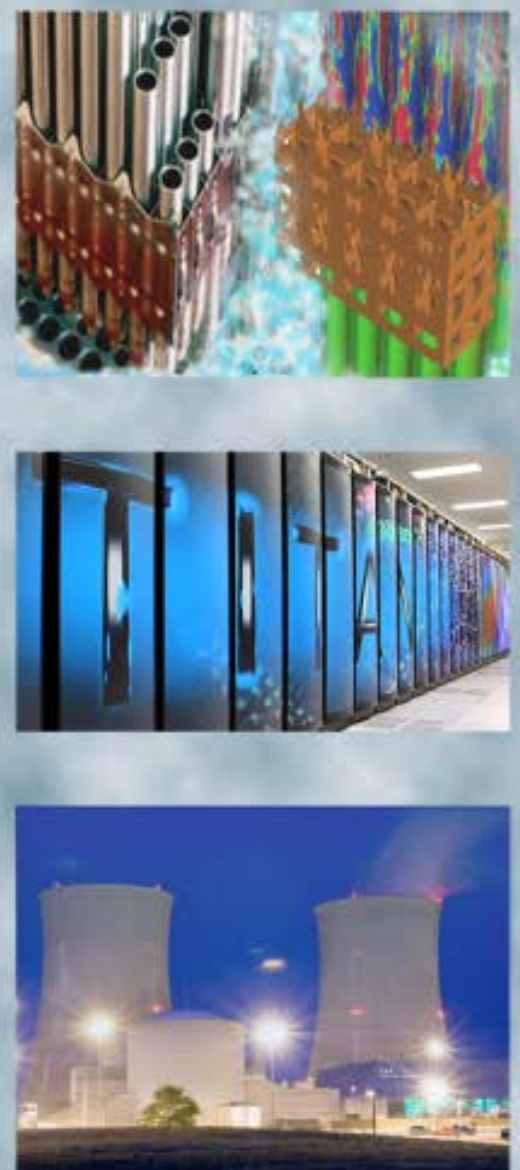
Please complete sections appropriate for this record.

REVISION LOG

\begin{tabular}{|c|c|c|l|}
\hline Revision & Date & Affected Pages & \multicolumn{1}{c|}{ Revision Description } \\
\hline 0 & $01 / 17 / 2017$ & All & Initial Release \\
\hline & & & \\
\hline & & & \\
\hline & & & \\
\hline
\end{tabular}

\section{Document pages that are:}

Export Controlled NONE

IP/Proprietary/NDA Controlled_NONE

Sensitive Controlled_NONE

Unlimited: ALL

\section{Requested Distribution:}

To:

Copy:

This report was prepared as an account of work sponsored by an agency of the United States Government. Neither the United States Government nor any agency thereof, nor any of their employees, makes any warranty, express or implied, or assumes any legal liability or responsibility for the accuracy, completeness, or usefulness of any information, apparatus, product, or process disclosed, or represents that its use would not infringe privately owned rights. Reference herein to any specific commercial product, process, or service by trade name, trademark, manufacturer, or otherwise, does not necessarily constitute or imply its endorsement, recommendation, or favoring by the United States Government or any agency thereof. The views and opinions of authors expressed herein do not necessarily state or reflect those of the United States Government or any agency thereof. 
MOOSE Implementation of MAMBA

Christopher Matthews, Jack Galloway

Los Alamos National Laboratory

The development of MAMBA is targeted at capturing both core wide CRUD induced power shifts (CIPS) as well as pin-level CRUD induced localized corrosion (CILC). Both CIPS and CILC require some sort of information from thermal-hydraulic, neutronics, and fuel performance codes, although the degree of coupling is different for the two effects. Since CIPS necessarily requires a core-wide power distribution solve, it requires tight coupling with a neutronics code. Conversely, CIPS tends to be an individual pin phenomenon, requiring tight coupling a fuel performance code. As efforts are now focused on coupling MAMBA within the VERA suite, a natural separation has surfaced in which a FORTRAN rewrite of MAMBA is optimal for VERA integration to capture CIPS behavior, while a CILC focused calculation would benefit from a tight coupling with BISON, motivating a MOOSE version of MAMBA.

The physical models incorporated within MAMBA tend to be calibrated coupled rate equations and a thermal solution. While these types of phenomena are relatively easy to implement in MOOSE, the meshing strategy that is utilized for CRUD growth does not easily translate to the finite-element framework that MOOSE is built upon. MAMBA currently uses a type of "fill-up" method in which only one node is active in the radial direction. As CRUD growth occurs (or losses occur due to etching by turbulent fluid flow) the material density within the node changes. Once the node reaches the peak density observed with CRUD (about 70\%), then the node is flagged as "filled." Subsequently, the radially adjacent empty node becomes "activated" and begins to fill. This method ensures that only one radial element is activated and is essentially a volume approximation to the surface growth. Due to the volume smearing of the crud growth, the fully coupled thermal-diffusion solve is limited to the mesh size.

In order to transition to MOOSE, an adequate meshing strategy has to be developed that captures the moving front of the CRUD layer, while allowing for higher fidelity simulations via fully coupled solves. Currently, the two methods that can be utilized in MOOSE are the extended finite element method (XFEM) or phase-field method (PFM). XFEM has recently been included in the MOOSE framework and historically has focused on crack growth, although it has previously been applied to sharp phase transitions and grain separations. However, due to the recent implementation into MOOSE, the method is not fully supported and will require significant method development to be applied to CRUD.

PFM has been extensively utilized in MOOSE primarily through the MARMOT code, and is naturally suited to the finite-element method through the utilization of diffuse boundaries. PFM has been applied to oxidation in zirconium cladding in the past (Figure 1 ), and shows promise with being able to capture not only CRUD growth, but also the interplay between the cladding/oxide/CRUD/water boundaries. 
The primary difficulty with the PFM lies in determination of adequate physical parameters; however the current version of MAMBA already requires tuning to experimental data. The PFM method provides ease with coupling to BISON through the MOOSE framework and will enable future physics to be implemented as necessary, such as mechanical interactions (which have been shown to be important to capture oxide growth) or two-phase chimney boiling. Mesh refinement already built into MOOSE may help accelerate solutions of the model; although methods may have to be developed to ensure the moving fronts are captured.

The implementation of a moving-front layer growth through the PFM, the CRUD and oxide layers can be calculated and used to inform full BISON fuel performance simulations. The flexibility of the PFM will also provide a framework to implement additional models as the understanding of CRUD increases over time.
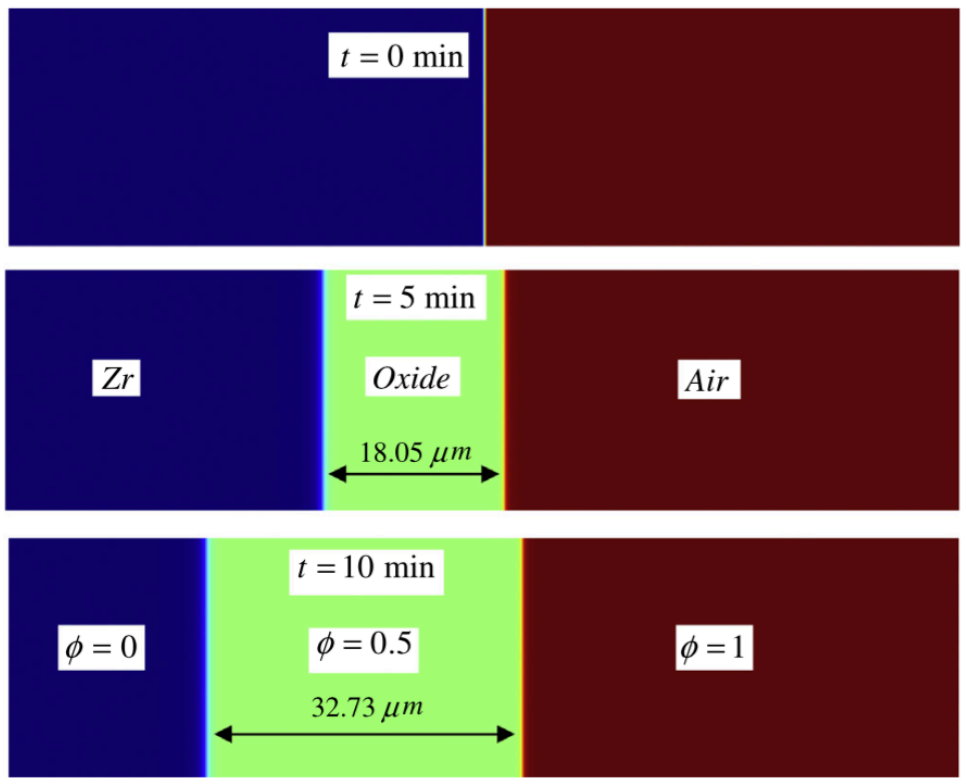

Figure 1: Oxide growth on zirconium cladding exposed to high temperature air (Zaeem \& Kadiri, JNM 89 2014). 